\title{
RETRACTIONS AND OTHER CONTINUOUS MAPS FROM $\beta X$ ONTO $\beta X \backslash X$
}

\author{
BY \\ W. W. COMFORT $\left({ }^{1}\right)$
}

SummaRY. Our two main theorems are stated below. The first is proved with the aid of the continuum hypothesis.

Theorem 2.6. $[\mathrm{CH}]$ Suppose that there is a retraction from $\beta X$ onto $\beta X \backslash X$. Then $X$ is locally compact and pseudocompact.

Theorem 4.2. Let $D$ be a discrete space whose cardinal number $\mathfrak{m}$ exceeds 1 . In order that there exist a continuous function from $\beta D$ onto $\beta D \backslash D$, it is necessary and sufficient that $\mathrm{m}=\mathrm{m}^{\mathrm{x}_{0}}$.

The proof of Theorem 2.6 rests on a result of Walter Rudin concerning $P$-points (see 1(d) and 1(e) below); Theorem 4.2 depends on the following simple result, which appears to be new.

TheOREM 4.1. Let $D$ be the discrete space with cardinal number $\mathfrak{m}\left(\geqq \boldsymbol{\aleph}_{0}\right)$. The smallest cardinal number which is the cardinal number of some dense subset of $\beta D \backslash D$ is $\mathrm{m}^{\aleph_{0}}$.

1. Introduction. Definitions, notation, discussion. Our chief source for background material, notation, terminology and helpful ideas is the GillmanJerison text [3].

The spaces we consider are all assumed to be completely regular Hausdorff spaces, and this fact will not be mentioned again. The collection of realvalued continuous functions on $X$ is denoted by the symbol $C(X)$, and the collection of bounded real-valued continuous functions on $X$ is denoted by the symbol $C^{*}(X)$. If $Y \subset X$ and each function in $C(Y)$ is the restriction to $Y$ of some function in $C(X)$, then we say that $Y$ is $C$-embedded in $X$; the expression " $Y$ is $C$ "-embedded in $X$ " is defined analogously.

For each space $X$ there is a compact space $\beta X$ containing (a homeomorph of) $X$ as a dense subset, with the property that any continuous function mapping $X$ into a compact space $Y$ admits a continuous extension (from $\beta X$ into $Y)$. In particular $X$ is $C^{*}$-embedded in $\beta X$; and $\beta X$ is, up to a homeomorphism leaving $X$ fixed pointwise, the only compact space in which $X$ is dense and $C^{*}$-embedded.

For later use we record here two well-known facts, which occur as 3.11(c) and $6.9(\mathrm{a})$ in [3].

Presented to the Society, August 25, 1964; received by the editors July 3, 1963.

(1) The author was supported in part by the National Science Foundation, under contract NSF-G23799. 
1(a) A compact space is $C$-embedded in any space containing it.

1(b) If $Y$ is $C^{*}$-embedded in $X$, then $\beta Y=\operatorname{cl}_{\beta X} Y$.

The space $X$ is said to be pseudocompact if $C^{*}(X)=C(X)$, i.e., if each real-valued continuous function on $X$ is bounded. We will use the following characterization of pseudocompact spaces, which is 1.21 of [3]:

1(c) A space is pseudocompact if and only if it contains no countably infinite $C$-embedded discrete subset.

Whenever we need to appeal to the continuum hypothesis to prove a theorem or corollary, we begin the formal statement of the result in question with the symbol $[\mathrm{CH}]$.

Following Gillman and Henriksen (in [2]) we shall say that a point $x$ in $X$ is a $P$-point in $X$ if each $G_{\delta}$ in $X$ containing $x$ is a neighborhood of $x$. An equivalent property is that each element of $C^{*}(X)$ is constant on some neighborhood of $x$. A $P$-space is by definition a space each of whose points is a $P$-point. Various characterizations of $P$-spaces are given in $4 \mathrm{~J}$ of $[3]$.

The following two assertions are due to Rudin (see [9, Theorem 4.5 ]; we have replaced the normality hypothesis imposed upon $X$ by Rudin (p. 633) with the weaker assumption that the discrete subset in question is $C^{*}$-embedded). Outlines of the proofs are available in $9 \mathrm{M}$ and $6 \mathrm{~V}$ of [3]; for our purposes it is necessary (and legitimate) to replace the expression " $C$-embedded" in $9 \mathrm{M} .2$ of [3] with the expression " $C$ *embedded closed."

1(d) Let $N$ be a countably infinite $C^{*}$-embedded closed discrete subset of the locally compact space $X$. Then $(\beta N \backslash N \subset \beta X \backslash X$ and) every $P$-point of $\beta N \backslash N$ is a $P$-point of $\beta X \backslash X$.

1(e) $[\mathrm{CH}]$ If $N$ is the countably infinite discrete space, then $\beta N \backslash N$ has a $P$-point.

It is a pleasure to thank the referee for an exceptionally detailed, careful and thoughtful criticism of this paper.

2. The retraction theorem. We approach the retraction theorem, which is 2.6, in easy steps.

2.1 THEOREM. Let $r$ be a continuous mapping from $X$ onto $Z$, and let $r$ be either open or closed. Let $Y$ be a dense subset of $X$, and suppose that whenever $x_{1}$ and $x_{2}$ are distinct points in $X$ for which $r x_{1}=r x_{2}$, then there is a point $y$ in $Y$ for which $r x_{1}=r y$. If $Y$ is $C^{*}$-embedded in $X$, then $r Y$ is $C^{*}$-embedded in $Z$; if $Y$ is $C$-embedded in $X$, then $r Y$ is $C$-embedded in $Z$.

Proof. We give only the $C^{*}$ proof. The reader may achieve the other by erasing the symbol * whenever it appears.

The symbol $r^{\prime}$ will denote the restriction of $r$ to $Y$. 
Let $f \in C^{*}(r Y)$, so that $f \circ r^{\prime} \in C^{*}(Y)$. There is a function $g$ in $C^{*}(X)$ for which $g(y)=\left(f \circ r^{\prime}\right)(y)$ whenever $y \in Y$. In a moment we will prove this assertion:

(a) if $x_{1} \in X$ and $x_{2} \in X$ and $r x_{1}=r x_{2}$, then $g x_{1}=g x_{2}$. Once (a) is proved we can legitimately define a real-valued function $\widetilde{f}$ on $Z$ by the relation

$$
\tilde{f}(r x)=g x
$$

and, having done so, complete the proof by showing

(b) $\widetilde{f}$ agrees with $f$ on $r Y$; and

(c) $\tilde{f} \in C^{*}(Z)$.

In proving (a), we may evidently suppose without loss of generality that $x_{1} \in Y$. Since $Y$ is dense in $X$, there is a net $y_{\alpha}$ in $Y$ for which $\lim _{\alpha} y_{\alpha}=x_{2}$. Then

$$
g x_{2}=\lim _{\alpha} g\left(y_{\alpha}\right)=\lim _{\alpha}\left(f \circ r^{\prime}\right)\left(y_{\alpha}\right) .
$$

Since $r$ is continuous on $X$ we have

$$
\lim _{\alpha} r\left(y_{\alpha}\right)=r x_{2}=r x_{1} \in r Y .
$$

But $f$ is continuous on $r Y$, so that

$$
\begin{aligned}
g x_{1} & =\left(f \circ r^{\prime}\right)\left(x_{1}\right)=f\left(r^{\prime} x_{1}\right)=f\left(r x_{1}\right) \\
& =f\left(\lim _{\alpha} r\left(y_{\alpha}\right)\right)=\lim _{\alpha} f\left(r\left(y_{\alpha}\right)\right) \\
& =\lim _{\alpha}(f \circ r)\left(y_{\alpha}\right)=\lim _{\alpha}\left(f \circ r^{\prime}\right)\left(y_{\alpha}\right) .
\end{aligned}
$$

For (b), choose any point $r y$ in $r Y$. Then

$$
\widetilde{f}(r y)=g y=\left(f \circ r^{\prime}\right)(y)=f\left(r^{\prime} y\right)=f(r y),
$$

so that $\tilde{f}$ agrees with $f$ on $r Y$.

To prove (c), we observe first that from (a) it follows that $\widetilde{f}^{-1}(A)=r\left(g^{-1} A\right)$ for each subset $A$ of $R$. Letting $A$ be an arbitrary open set if $r$ is open, and letting $A$ be an arbitrary closed set if $r$ is closed, we see that $\widetilde{f}$ is continuous on $Z$.

2.2 Theorem. Let $r$ be a continuous map from the compact space $X$ onto $Z$ and let $Y$ be $C^{*}$-embedded in $X$. Suppose that whenever $p_{1}$ and $p_{2}$ are distinct points in $\mathrm{cl}_{X} Y$ for which $r p_{1}=r p_{2}$, then there is a point $y$ in $Y$ for which $r p_{1}=r y$. Then $r Y$ is $C^{*}$-embedded in $Z$.

Proof. We let $\bar{X}=\operatorname{cl}_{X} Y, \bar{Y}=Y, \bar{r}=r \mid \bar{X}, \bar{Z}=\bar{r} \bar{X}$. Applying 2.1 to the - ed symbols we find that $\bar{r} \bar{Y}$, which is $r Y$, is $C^{*}$-embedded in $\bar{r} \bar{X}$. But $\bar{r} \bar{X}$, being compact, is $C^{*}$-embedded in $Z$ by 1 (a), and the theorem follows. 
2.3 THEOREM. Let $r$ be a continuous map from $\beta X$ onto the (compact) space $Z$, and let $r$ be one-to-one on $\beta X \backslash X$. Let $Y$ be a subset of $X$ that is $C^{*}$-embedded and closed (in $X)$. Then $r Y$ is $C^{*}$-embedded in $Z$, and $\beta(r Y)=r(\beta Y)$.

Proof. The first assertion follows from 2.2, and the second from the first as follows:

$$
\beta(r Y)=\operatorname{cl}_{Z} r Y=r\left(\operatorname{cl}_{\beta X} Y\right)=r(\beta Y) .
$$

2.4 Theorem. Let $r$ be a retraction from $\beta X$ onto $\beta X \backslash X$, and let $Y$ be a subset of $X$ that is $C^{*}$-embedded and closed (in $X$ ). Then $r Y$ is $C^{*}$-embedded in $\beta X \backslash X$, and $\beta(r Y)=r(\beta Y)$. In particular, $\beta(r X)=\beta X \backslash X$.

Proof. Choose $Z=\beta X \backslash X$.

To prove our next result, we shall use the continuum hypothesis.

2.5 Theorem. [ $\mathrm{CH}]$ Suppose that there is a retraction from $\beta X$ onto $\beta X \backslash X$. Then $X$ is locally compact, and $X$ admits no countably infinite $C^{*}$-embedded closed discrete subset.

Proof. Denoting by $r$ the hypothesized retraction, we have $r(\beta X)=\beta X \backslash X$, so that $X$ is open in $\beta X$. Hence $X$ is locally compact.

If $M$ is a countably infinite $C^{*}$-embedded closed discrete subset of $X$, then from 2.4 we have

$$
\beta(r M)=r(\beta M)=r M \cup(\beta M \backslash M) .
$$

This set, the closure in $\beta X$ of $r M$, contains $\beta M \backslash M$. Since $\beta M \backslash M$ has $2^{c}$ points (see Theorem 1.3 of [9] or Theorem 9.2 of [3]), the set $r M$ is not finite. We claim a little more: $r M \backslash(\beta M \backslash M)$ is infinite. This is true because otherwise the countable set $r M \cap(\beta M \backslash M)$ would be dense in $\beta M \backslash M$, contrary to $6 \mathrm{Q} .2$ of [3] or Theorem 4.1 of [1]. Consequently we may choose a countably infinite discrete subset $K=\left\{p_{1}, p_{2}, \cdots\right\}$ of $r M \backslash(\beta M \backslash M)$. Next for each positive integer $n$ we choose a point $x_{n}$ in $r^{-1}\left(p_{n}\right) \cap M$. Defining $N=\left\{x_{n}\right\}_{n=1}^{\infty}$, we conclude the proof by making six observations. Of these, (1) is obvious and (together with 2.4 and the fact that $K \cap(\beta N \backslash N)$ $\neq \emptyset)$ implies (2), from which (3) follows; (5) follows from (4), which is $1(\mathrm{~d})$; (6), which is with (5) the desired contradiction, is a consequence of (2). The observations (1) through (6) are true as stated below independent of the continuum hypothesis. The continuum hypothesis is used because without it we do not know how to deduce the existence of a $P$-point in $\beta N \backslash N$.

(1) $N$ is a countably infinite $C^{*}$-embedded closed discrete subset of $X$;

(2) $K$ is $C^{*}$-embedded in $\beta X \backslash X$, and $\beta N \backslash N=\beta K \backslash K$;

(3) the function $f$, defined on $K$ by the relation $f\left(p_{n}\right)=1 / n$, has a continuous extension $\widetilde{f}$ to $\beta X$, and $\widetilde{f}$ vanishes on $\beta N \backslash N$;

(4) if $p$ is a $P$-point of $\beta N \backslash N$, then $p$ is a $P$-point of $\beta X \backslash X$; 
(5) there is a neighborhood in $\beta X \backslash X$ of $p$ on which $\tilde{f}$ vanishes;

(6) each neighborhood of $p$ meets $K$.

2.6 Theorem. $[\mathrm{CH}]$ Suppose that there is a retraction from $\beta X$ onto $\beta X \backslash X$. Then $X$ is locally compact and pseudocompact.

Proof. From 1(c) and 2.5.

REmarks. The theorem above is strictly weaker than Theorem 2.5 , for Katětov has given in [6] an example of a locally compact pseudocompact space one of whose countably infinite closed discrete subspaces is $C^{*}$ embedded. Details are in $6 \mathrm{P}$ of [3].

The author's attempts to prove Theorem 2.6 without the continuum hypothesis have been successful only for $F$-spaces. These spaces, which have been studied and given numerous characterizations in Chapter 14 of [3], may be defined as those spaces $X$ for which every finitely generated ideal in the ring $C^{*}(X)$ is principal.

2.7 Theorem. Suppose there is a retraction from $\beta X$ onto $\beta X \backslash X$. Then $X$ is locally compact; and if $X$ is an $F$-space, then $X$ admits no countably infinite $C^{*}$-embedded closed discrete subset.

Proof. Assuming otherwise, construct $K$ and $N$ as in the proof of 2.5. Since $K$ misses the compact set $N \cup(\beta N \backslash N)$, and $N$ misses the compact set $K \cup(\beta N \backslash N)$, both $N$ and $K$ are (relatively) open subsets of the space $N \cup K$. The function on $N \cup K$ which is 1 on $K$ and 0 on $N$ is consequently continuous, but it evidently admits no continuous extension to any point of $\beta N \backslash N$. This is ridiculous since $\beta X$, like $X$, is an $F$-space, and each countable subset of any $F$-space is $C^{*}$-embedded [3, 14N.5].

2.8 CoRollary. If $X$ is a nonempty $P$-space there is no retraction from $\beta X$ onto $\beta X \backslash X$.

Proof. Like every $P$-space, $X$ is an $F$-space. If the corollary fails, then $X$ is pseudocompact by 2.7 ; but a pseudocompact $P$-space is finite $[3,4 \mathrm{~K} .2]$, so that $\beta X \backslash X$ is empty.

We conclude this section with a result in the spirit of 2.5 . The property imposed upon $X$, and its relation to other topological properties, has been studied by Marczewski in [8].

2.9 Theorem. Let $X$ be a topological space which admits no uncountable collection of pairwise disjoint nonempty open subsets, and suppose that there is a continuous function $f$ mapping $\beta X$ onto $\beta X \backslash X$. Then $X$ is pseudocompact.

Proof. Hugh Gordon and the author have shown in [1] that if $X$ is locally compact but not pseudocompact, then there is a collection $\mathscr{U}$ of $c$ pairwise disjoint nonempty open subsets of $\beta X \backslash X$. To obtain a contradiction, we confront the hypotheses with the collection $\left\{X \cap f^{-1} U \mid U \in \mathscr{U}\right\}$. 
3. Corollaries and examples. The realcompact spaces mentioned below are the $Q$-spaces introduced by Hewitt in [5]. A space is realcompact if and only if it is homeomorphic with a closed subset of a product of real lines (see [3, Chapters 8 and 11]).

We shall say that a space $X$ has the retraction property if there exists a retraction from $\beta X$ onto $\beta X \backslash X$.

3.1 Corollary. Let $X$ be nonempty.

(a) If $X$ is realcompact and separable, then there is no continuous function from $\beta X$ onto $\beta X \backslash X$.

(b) $[\mathrm{CH}]$ If $X$ is realcompact or metrizable, then $X$ does not have the retraction property.

Proof. If (a) fails, then $X$ is pseudocompact by 2.9. If (b) fails, then $X$ is pseudocompact by 2.6. But a pseudocompact space, if it is realcompact or metrizable, is compact (see $[3,5 \mathrm{H} .2$ and $3 \mathrm{D} .2]$ ). If either (a) or (b) fails, then there is a mapping from $\beta X$ to $\beta X \backslash X=\emptyset$, which is obsurd.

3.2 Corollary. Let $X$ be normal, and let $r$ be any retraction from $\beta X$ onto $\beta X \backslash X$. Then $r X$ is normal.

Proof. We recall from 3D.1 of [3] that a space is normal if and only if each of its closed subsets is $C^{*}$-embedded. If $A$ is closed in $r X$, then $\left(r^{-1} A\right) \cap X$ is closed in $X$. The result now follows from 2.3 above, once it is observed that $A=r\left(r^{-1} A \cap X\right)$.

3.3 Corollary. Suppose that $\beta X \backslash X$ is an absolute retract for compact spaces. Then $X$ is pseudocompact.

Proof. Trivial from 2.6. For an alternative proof, not dependent upon the continuum hypothesis, suppose that $X$ is not pseudocompact and let $\mathscr{U}$ be a collection of $\mathfrak{c}$ pairwise disjoint nonempty open subsets of $\beta X \backslash X$, as afforded by Corollary 3.2 of [1]. If $Y$ is a product of closed intervals containing (a homeomorph of ) $\beta X \backslash X$, and if $r$ is a retraction of $Y$ onto $\beta X \backslash X$, then the family $\left\{r^{-1} U \mid U \in \mathscr{U}\right\}$ of pairwise disjoint nonempty open subsets of $Y$ is uncountable, contrary to Marczewski's Theorem 3.3 in [8].

Example 3.5 below shows that the product of two spaces, each with the retraction property, may fail to have the retraction property. I do not know whether there are two nonempty spaces with the retraction property whose product also has the retraction property. If he is interested in this question, the reader will wish to recall from [4] the following special case of Glicksberg's Theorem 3: In order that the product of two pseudocompact spaces be pseudocompact, it is sufficient that one of the spaces be locally compact. 
3.4 Theorem. [ $\mathrm{CH}]$ If $X$ has the retraction property and $Y$ is compact, then $X \times Y$ has the retraction property.

Proof. That $X \times Y$ is pseudocompact follows from our 2.6 and the Glicksberg result just quoted. Hence $\beta(X \times Y)=\beta X \times \beta Y=\beta X \times Y$ by Theorem 1 of [4]. If $r$ is the hypothesized function, then $(p, q) \rightarrow(r p, q)$ is the required mapping defined on $\beta(X \times Y)$.

It is convenient, or at least conventional, to distinguish notationally between the smallest uncountable ordinal and the set of all countable ordinals. We denote these spaces, the latter supplied with the usual order topology, by the symbols $\omega_{1}$ and $W$ respectively. The set of ordinals not exceeding $\omega_{1}$, when given its order topology, is (homeomorphic with) $\beta \mathbf{W}$; it is $\mathbf{W} \cup\left\{\omega_{1}\right\}$, and it will be denoted by the symbol $\mathbf{W}^{*}$. The set $\mathbf{W}^{*} \backslash \mathbf{W}$ has exactly one point; hence $W$ has the retraction property.

3.5 ExAMPLE. $\mathbf{W} \times \mathbf{W}$ does not have the retraction property.

Proof. From the Glicksberg theorem used in the proof of 3.4 we have $\beta(\mathbf{W} \times \mathbf{W})=\mathbf{W}^{*} \times \mathbf{W}^{*}$. We denote by $E$ and $T$ the sets $\left\{\omega_{1}\right\} \times \mathbf{W}$ and $\mathbf{W} \times\left\{\omega_{1}\right\}$ respectively, so that $\left(\omega_{1}, \omega_{1}\right) \notin E \cup T$. Assuming the existence of a retraction $r$, we proceed by adopting an idea from $4 \mathrm{E}$ of [7].

Let $x_{1}<\omega_{1}$ and note that the point $\left(\omega_{1}, x_{1}\right)$, which is $r\left(\omega_{1}, x_{1}\right)$, does not belong to the closed set $T \cup\left\{\left(\omega_{1}, \omega_{1}\right)\right\}$. Thus there exists $x_{2}$, with $x_{1}<x_{2}<\omega_{1}$, for which $r\left(x_{2}, x_{1}\right) \in E$. Reasoning similarly we can find an ordinal $x_{3}$, with $x_{2}<x_{3}<\omega_{1}$, for which $r\left(x_{2}, x_{3}\right) \in T$. In general, $x_{n}$ having been chosen whenever $n<2 k$, we choose $x_{2 k}$ so that $x_{2 k-1}$ $<x_{2 k}<\omega_{1}$ and $r\left(x_{2 k}, x_{2 k-1}\right) \in E$; then we choose $x_{2 k+1}$ so that $x_{2 k}<x_{2 k+1}$ $<\omega_{1}$ and $r\left(x_{2 k}, x_{2 k+1}\right) \in T$. It is easy to see, setting $p=\sup x_{n}$, that

$$
\lim _{k}\left(x_{2 k}, x_{2 k-1}\right)=\lim _{k}\left(x_{2 k}, x_{2 k+1}\right)=(p, p) .
$$

Thus $r(p, p) \in \operatorname{cl} T \cap \operatorname{cl} E$, so that $r(p, p)=\left(\omega_{1}, \omega_{1}\right)$. It follows that the sequence $r\left(x_{2 k}, x_{2 k-1}\right)$, which is a countable sequence in $E$, has limit $\left(\omega_{1}, \omega_{1}\right)$; but this is impossible because $E$ is homeomorphic with $\mathbf{W}$, and so no countable subset of $E$ is cofinal in $E$ (see $5 E(e)$ of [7] or 5.12(a) of [3]).

3.6 REMARK. The example just given shows that the converse to 2.6 is false: $\mathbf{W} \times \mathbf{W}$ is a locally compact, pseudocompact space that does not enjoy the retraction property. For a simpler example of this phenomenon, consider the so-called "long line" (which, because it is "long" in only one direction, might more appropriately be called the "long ray"). This space is homeomorphic with the space $\mathbf{W} \times[0,1]$, lexicographically ordered. We identify a point (the first point, say) from each of two disjoint copies of this space, so that the resulting space, which we call $\mathbf{L}$, is connected. Then $\beta \mathbf{L} \backslash \mathbf{L}$, which is the discrete space with two points, is not a retract of the connected space $\beta \mathbf{L}$. 
In the following example we use the space $\mathbf{L}$ to show that the retraction property need not be preserved under retraction.

3.7 Example. There is a space $X$, and a retraction $r$ from $\beta X$ onto $\beta X \backslash X$, with the following properties:

(a) $r X$ is a locally compact proper subset of $\beta X \backslash X$;

(b) $r X$ does not have the retraction property.

Proof. Let $X=\beta \mathbf{L} \times \mathbf{W}$. Then, again by Theorem 1 of [4], we have $\beta X=\beta \mathbf{L} \times \mathbf{W}^{*}$. One available retraction from $\beta X$ onto $\beta X \backslash X$ is the map $(\alpha, y) \rightarrow\left(\alpha, \omega_{1}\right)$. In defining an alternative retraction we shall adjoin the subscript $p$ or $n$ to each nonzero point of $\mathbf{L}$, according as the point lies in the "positive" or "negative" of those two long rays of whose union $\mathbf{L}$ is a quotient space. The retraction taking $\beta X$ onto $\beta X \backslash X$ is defined on $X$ as follows:

$$
\begin{aligned}
& r((0,0), y)=\left((0,0), \omega_{1}\right), \\
& r\left((x, t)_{p}, y\right)= \begin{cases}\left((y, 0)_{p}, \omega_{1}\right) & \text { if } \omega_{1}>x \geqq y, \\
\left((x, t)_{p}, \omega_{1}\right) & \text { if } x<y,\end{cases} \\
& r\left((x, t)_{n}, y\right)= \begin{cases}\left((y, 0)_{n}, \omega_{1}\right) & \text { if } \omega_{1}>x \geqq y, \\
\left((x, t)_{n}, \omega_{1}\right) & \text { if } x<y,\end{cases} \\
& r\left(\left(\omega_{1}\right)_{p}, y\right)=\left((y, 0)_{p}, \omega_{1}\right), \\
& r\left(\left(\omega_{1}\right)_{n}, y\right)=\left((y, 0)_{n}, \omega_{1}\right) .
\end{aligned}
$$

We have $r X=\mathbf{L} \times\left\{\omega_{1}\right\} \subsetneq \beta \mathbf{L} \times\left\{\omega_{1}\right\}=\beta X \backslash X$, and 3.6 shows that $r X$ does not have the retraction property.

4. Continuous mappings from $\beta D$ onto $\beta D \backslash D$. The density character of the topological space $Y$ is the smallest cardinal number which is the cardinal number of some dense subset of $Y$; it is denoted by the symbol $\delta(Y)$.

4.1 THEOREM. Let $D$ be the discrete space with cardinal number $m\left(\geqq \boldsymbol{\aleph}_{0}\right)$. Then $\delta(\beta D \backslash D)=\mathfrak{m}^{\aleph_{0}}$.

Proof. It is known (see [1, Theorem 4.1]) that $\beta D \backslash D$ admits a collection of $\mathfrak{m}^{\mathrm{N}_{0}}$ pairwise disjoint nonempty open subsets. Since any dense subset of $\beta D \backslash D$ must meet each of these sets, we have $\delta(\beta D \backslash D) \geqq \mathfrak{m}^{\boldsymbol{x}_{0}}$.

For the reverse inequality, let $\mathscr{C}$ denote the collection of all countably infinite subsets of $D$, and for each $N$ in $\mathscr{C}$ choose a point $p_{N}$ in $\left(\operatorname{cl}_{\beta D} N\right) \backslash D$. (This is possible because $N$ is not closed in $\beta D$.) It is easy to see, arguing as in $6 \mathrm{~S}$ of [3], that any open subset $U$ of $\beta D \backslash D$ contains a set of the form $\left(\mathrm{cl}_{\beta D} E\right) \backslash D$, where $E$ is an infinite subset of $D$. Then, choosing $N \in \mathscr{C}$ with $N \subset E$, we have $p_{N} \in U$. Thus the set $\left\{p_{N} \mid N \in \mathscr{C}\right\}$, whose cardinality does not exceed $\mathrm{m}^{\boldsymbol{\aleph}_{0}}$, is dense in $\beta D \backslash D$. 
4.2 Theorem. Let $D$ be a discrete space whose cardinal number $\mathfrak{m}$ exceeds 1 . In order that there exist a continuous function from $\beta D$ onto $\beta D \backslash D$, it is necessary and sufficient that $\mathfrak{m}=\mathfrak{m}^{\boldsymbol{N}_{0}}$.

Proof. When $m$ is finite the two conditions, being false, are equivalent.

NeCEssity. If $f$ is the hypothesized function onto $\beta D \backslash D$, then $f D$ is dense in $\beta D \backslash D$, so that $\delta(\beta D \backslash D) \leqq \mathfrak{m}$.

SuFficiency. Let $E$ be a dense subset of $\beta D \backslash D$ for which card $E=\mathfrak{m}^{\aleph_{0}}$ $=\mathfrak{m}$, and let $f$ be any function mapping $D$ onto $E$. The Stone-Čech extension property guarantees that there is a continuous function $\widetilde{f}$, agreeing with $f$ on $D$ and mapping $\beta D$ into $\beta D \backslash D$. The range of $\widetilde{f}$ is compact and dense in $\beta D \backslash D$, so that $\widetilde{f}$ is onto.

To deduce our final result from 4.2 , we need only observe that $\aleph_{1}^{\aleph_{0}}=c$.

4.3 Corollary. Let $D$ be the discrete space for which card $D=\aleph_{1}$. Then the following assertions are equivalent:

(a) there is a continuous function from $\beta D$ onto $\beta D \backslash D$;

(b) the continuum hypothesis is valid.

\section{REFERENCES}

1. W. W. Comfort and H. Gordon, Disjoint open subsets of $\beta X \backslash X$, Trans. Amer. Math. Soc. 115 (1964); 513-520.

2. L. Gillman and M. Henriksen, Concerning rings of continuous functions, Trans. Amer. Math. Soc. 77 (1954), 340-362.

3. L. Gillman and M. Jerison, Rings of continuous functions, Van Nostrand, Princeton, N. J., 1960.

4. I. Glicksberg, Stone-Čech compactifications of products, Trans. Amer. Math. Soc. 90 (1959), 369-382.

5. E. Hewitt, Rings of real-valued continuous functions. I, Trans. Amer. Math. Soc. 64 (1948), 45-99.

6. M. Katětov, On real-valued functions in topological spaces, Fund. Math. 38 (1951), 85-91.

7. J. L. Kelley, General topology, Van Nostrand, New York, 1955.

8. E. Marczewski, Séparabilité et multiplication Cartésienne des espaces topologiques, Fund. Math. 34 (1947), 127-143.

9. W. Rudin, Homogeneity problems in the theory of Cech compactifications, Duke Math. J. 23 (1956), 409-419, 633.

\section{UNIVERSITY OF ROCHESTER, ROCHESTER, NEW YORK}

